\title{
BMJ Open Systematic review of percutaneous closure versus medical therapy in patients with cryptogenic stroke and patent foramen ovale
}

\author{
Frederick A Spencer, ${ }^{1}$ Luciane C Lopes, ${ }^{2}$ Sean A Kennedy, ${ }^{3}$ Gordon Guyatt ${ }^{4}$
}

To cite: Spencer FA, Lopes LC, Kennedy SA, et al. Systematic review of percutaneous closure versus medical therapy in patients with cryptogenic stroke and patent foramen ovale. BMJ Open 2014;4:e004282. doi:10.1136/bmjopen-2013004282

- Additional material is available. To view please visit the journal (http://dx.doi.org/ 10.1136/bmjopen-2013004282).

Received 18 October 2013 Revised 28 January 2014 Accepted 10 February 2014

CrossMark

\author{
1Department of Medicine, \\ Mcmaster University, \\ Hamilton, Ontario, Canada \\ 2University of Sorocaba, \\ UNISO, Sorocaba, \\ Sao Paulo, Brazil \\ ${ }^{3}$ Mcmaster University, \\ Hamilton, Ontario, Canada \\ ${ }^{4}$ Department of Clinical \\ Epidemiology and \\ Biostatistics, McMaster \\ University, Hamilton, \\ Ontario, UK
}

Correspondence to Dr Frederick A Spencer; fspence@mcmaster.ca

\section{ABSTRACT}

Objectives: To provide a comprehensive comparison of patent foramen ovale (PFO) closure versus medical therapy in patients with cryptogenic stroke or transient ischaemic attack (TIA) and demonstrated PFO.

Design: Systematic review with complete case metaanalysis and sensitivity analyses. Data sources included MEDLINE and EMBASE from 1980 up to May 2013. All randomised controlled trials (RCTs) comparing treatment with percutaneous catheter-based closure of PFO to anticoagulant or antiplatelet therapy in patients with cryptogenic stroke or TIA and echocardiographically confirmed PFO or atrial septal defect (ASD) were eligible. Participants: 1967 participants with prior stroke or TIA and echocardiographically confirmed PFO or ASD.

Primary outcome measures: The primary outcome of interest was recurrence of ischaemic stroke. We utilised data from complete cases only for the primary endpoint and combined data from trials to estimate the pooled risk ratio (RR) and associated $95 \%$ Cls calculated using random effects models.

Results: We identified 284 potentially eligible articles of which three RCTs including 2303 patients proved eligible and 1967 patients had complete data. Of the 1026 patients randomised to PFO closure and followed to study conclusion 22 experienced non-fatal ischaemic strokes, as did 34 of 941 patients randomised to medical therapy (risk ratio (RR) $0.61,95 \% \mathrm{Cl} 0.34$ to 1.07 ; heterogeneity: $p=0.34, I^{2}=8 \%$, confidence in estimates low due to risk of bias and imprecision). Analyses for ischaemic stroke restricted to 'per-protocol' patients or patients with concomitant atrial septal aneurysm did not substantially change the observed RRs. Complication rates associated with either PFO closure or medical therapy were low.

Conclusions: Pooled data from three RCTs provides insufficient support that PFO closure is preferable to medical therapy for secondary prevention of cryptogenic stroke in patients with PFO.

\section{INTRODUCTION}

Observational studies suggest that younger patients with cryptogenic stroke are more likely to have a patent foramen ovale (PFO) than the general population. ${ }^{12}$ A proposed

\section{Strengths and limitations of this study}

- Estimation of absolute benefits and risks of treatment strategies.

- Careful assessment of risk of bias of individual studies using Cochrane criteria.

- Evaluation of overall confidence in pooled outcome(s) estimates using GRADE.

- Primary analysis was restricted to patients with available data (complete case analysis). If event rates differed in those with missing data in intervention and control groups-of particular concern would be higher rates of events in those lost to follow-up in the PFO closure arm than the medical therapy arm-the complete case results may be misleading.

- Individual patient-level data not available.

mechanism for stroke in these patients is passage of thrombi from the venous circulation to the arterial circulation through the PFO. Although what proportion of cryptogenic strokes are due to paradoxical embolism remains unknown, percutaneous closure of PFO using devices approved for haemodynamically significant secundum atrial septal defect (ASD) has increased greatly in the past two decades. A systematic review of observational studies suggests that PFO closure may be superior to medical therapy (antiplatelet or anticoagulant agents) for secondary prevention of stroke in patients with PFO and cryptogenic stroke. ${ }^{3}$

In the past 2 years three randomised controlled trials (RCTs) comparing PFO closure to medical therapy have been publishednone showed PFO closure to be statistically superior to medical therapy for the primary composite outcome but each reported trends favouring PFO closure. ${ }^{4-6}$ In one study, PFO closure was superior to medical therapy for the prevention of recurrent neurological events in prespecified per protocol and as-treated analyses. ${ }^{5}$ 
One systematic review and meta-analysis that included the three RCTs, and a second meta-analysis, have addressed this issue. Both were limited, however, by failure to fully consider risk of bias issues, failure to use the GRADE approach to determine overall confidence in estimates of intervention effect, and failure to consider the limitations of composite endpoints.

We, therefore, undertook a systematic review of all RCTs comparing percutaneous PFO closure to medical therapy in patients with cryptogenic stroke or transient ischaemic attack (TIA) and PFO or ASD. As composite endpoints varied between trials, we focused on individual endpoints of recurrent non-fatal stroke, recurrent TIA, death, major bleeding and atrial fibrillation. We also examined per protocol rates of recurrent stroke in patients undergoing PFO closure compared with the medical therapy arm. Outcomes were defined as in each study.

\section{METHODS}

\section{Eligibility criteria}

We included all RCTs comparing treatment with percutaneous catheter-based closure of PFO to medical therapy (anticoagulant or antiplatelet therapy) in patients with cryptogenic stroke or TIA and echocardiographically confirmed PFO or ASD. We excluded trials including participants with other indications for $\mathrm{PFO} / \mathrm{ASD}$ closure (eg, haemodynamic significance) or other indications for anticoagulant therapy (eg, atrial fibrillation).

Included articles met two prespecified criteria: (1) RCTs that compared PFO closure with medical therapy (antiplatelet or anticoagulant agents); (2) Greater than $90 \%$ of patients had prior unexplained stroke, TIA or other arterial embolism, or this subset was reported separately. When more than one study reported data from a population, we used the most complete and updated results.

\section{Data sources and search strategy}

We searched MEDLINE and EMBASE from 1980 to May 2013. We restricted the search to human participants. Keywords included PFO or ASD. Results were then limited to RCT or controlled clinical trial or phase 3 clinical trial or phase 4 clinical trial. For every eligible study we identified, and for studies such as review articles that included citations to potentially eligible studies, one reviewer examined the reference list.

\section{Study selection}

Teams of two investigators independently screened each title and abstract from this search. If either of the two screeners identified a citation as potentially relevant, we obtained the full text article for detailed review. Teams of two reviewers independently determined the eligibility of all studies that underwent full text evaluation. Disagreements were resolved through discussion between the two reviewers.

\section{Data abstraction}

Using a custom-made data collection form two of three reviewers (FAS, LCL, SAK) abstracted the following information from each identified study: mean follow-up time, total patient years follow-up (overall and per cohort), number of patients withdrawn or lost to follow-up, number of patients crossing over from medical therapy to PFO closure, number of patients undergoing PFO closure attempt, number of patients in whom PFO closure was technically successful, procedural complications (other than major bleeding) from PFO closure and outcome event rates.

Disagreements regarding data abstraction results were resolved through discussion between the two reviewers. The primary author abstracted additional information on study funding, eligibility criteria, patient demographics and treatment characteristics.

\section{Risk of bias and confidence in effect assessment}

Two reviewers (FAS, LCL) independently assessed, using the Cochrane risk for bias tool, seven domains: adequacy of sequence generation, allocation sequence concealment, blinding of participants and caregivers, blinding for outcome assessment, incomplete outcome data, selective outcome reporting, and the presence of other potential sources of bias not accounted for in the other six domains. ${ }^{7}$

We used GRADE methodology to rate confidence in estimates of effect for each outcome as high, moderate, low or very low. ${ }^{8}$ We used detailed GRADE guidance to assess overall risk of bias, ${ }^{9}$ imprecision, ${ }^{10}$ inconsistency, ${ }^{11}$ indirectness ${ }^{12}$ and publication bias ${ }^{13}$ and summarised results in an evidence profile.

For decisions regarding eligibility, risk of bias assessment and data abstraction, reviewers resolved disagreement through discussion.

\section{Data synthesis and statistical analysis}

We report descriptive statistics as proportions for categorical variables, and mean/median for continuous variables. Our primary analyses for non-fatal ischaemic stroke, TIAs and mortality included only patients with complete follow-up. We excluded patients for whom complete data was unavailable: those lost to follow-up, those who withdrew consent or those who were found to not have PFO/ASD or history of TIA/stroke after randomisation. For outcomes of bleeding and atrial fibrillation we used data as reported by the investigators from the intention to treat analyses.

Rates of non-fatal ischaemic stroke in patients treated as 'per-protocol' from two of the three studies were also abstracted and pooled. The definition of per-protocol varied from study to study (CLOSURE 1: all randomised patients who received the treatment to which they were randomised, who had no major inclusion/exclusion criteria violations and who had a follow-up of at least 22 months; RESPECT: patients who received the randomly assigned treatment, adhered to the protocol- 
mandated medical treatment, and did not have a major inclusion or exclusion violation). Event rates for nonfatal ischaemic stroke in the 'per-protocol' subset were not reported in PC Trial manuscript. The primary author of the manuscript did not respond to email requests for further information.

As previous observational studies suggest that patients with cryptogenic stroke and PFO may be at higher risk for recurrent stroke if they have a concomitant atrial septal aneurysm, we performed an additional complete case analysis for a primary composite outcome in the subset of patients with atrial septal aneurysm (data on non-fatal ischaemic stroke alone not reported in any of the studies for this subset). Composite outcomes in the three studies included some combination of non-fatal ischaemic stroke, TIA, peripheral embolism and death.

As two of the three studies used the Amplatzer device and one used the STARFlex device (CLOSURE 1), we conducted a sensitivity analysis for non-fatal ischaemic stroke excluding the CLOSURE study. We evaluated for subgroup difference (2 Amplatzer studies vs STARFlex study) using a $\chi^{2}$ test.

Given the high rate of patients excluded from complete case analyses (most due to loss to follow-up) we also conducted two additional analyses: (1) worst-case scenario in which we assumed that all patients with missing data in the PFO closure arms suffered non-fatal ischaemic strokes and all patients lost to follow-up in the medical arms did not and (2) plausible worst-case scenario in which all patients with missing data from the PFO closure arm were assumed to have five times the rate of stroke as the complete cases and those excluded from the medical therapy arm were assumed to have $1 / 5$ times the rate of stroke as the complete cases. ${ }^{14}$

We calculated pooled risk ratios (RRs) and associated 95\% CI for non-fatal ischaemic stroke and TIAs using random effects models applying Mantel-Haenszel method. Absolute effects (and 95\% CI) were calculated by multiplying pooled RRs and $95 \%$ CI by pooled control rate of outcomes. As event rates were very low for death, atrial fibrillation and major bleeding (leading to skewed 95\% $\mathrm{CI}$ ), pooled risk difference (RD) and $95 \% \mathrm{CI}$ was used to calculate absolute effects for these outcomes. ${ }^{15}$ Statistical heterogeneity was assessed by the $\mathrm{I}^{2}$ statistic. Analyses were performed using RevMan V.5.2 (Copenhagen: Nordic Cochrane Center, Cochrane Collaboration).

\section{RESULTS}

\section{Trial identification}

Our search yielded 284 abstracts-all were identified from the electronic database search of which 47 were duplicates and excluded. We excluded an additional 229 articles based on a review of the title and abstract, leaving 8 articles for full review. Of these studies, 5 were excluded-2 were descriptions of methodology for subsequently reported RCTs, 1 was a comparison of different devices for closure but did not include a medical therapy arm and 2 were prospective cohort studies of PFO closure (see online supplementary appendix figure). We included three randomised trials enrolling 2303 patients. ${ }^{4-7}$

\section{Trial and patient characteristics}

Table 1 presents the characteristics of the three eligible studies. Two of the three studies (RESPECT, PC Trial) used the Amplatzer occluder device, whereas the other used the STARFlex device (CLOSURE I). Crossover from medical therapy to PFO closure occurred in only one study $(13.3 \%$, PC Trial). The percentage of patients in the PFO closure arm undergoing a PFO closure attempt ranged from $90.6 \%$ to $96.1 \%$ with success rates ranging from $89.4 \%$ to $99.1 \%$.

In the CLOSURE I study, all patients undergoing PFO closure were assigned to clopidogrel $75 \mathrm{mg} /$ day for 6 months and aspirin 81 or $325 \mathrm{mg}$ /day for 2 years. In the RESPECT study, all patients undergoing closure received aspirin 81-325 mg plus clopidogrel for 1 month followed by aspirin monotherapy for 5 months. Antiplatelet treatment was thereafter left to the discretion of the site investigator. In the PC Trial, managing clinicians were counselled to recommend aspirin $100-325 \mathrm{mg}$ /day for 5-6 months and ticlopidine $(250-500 \mathrm{mg} /$ day $)$ or clopidogrel $(75-150 \mathrm{mg} /$ day $)$ for $1-6$ months. However, at discharge from PFO closure in the PC Trial, 182 patients (89.2\%) were using aspirin, $104(51 \%)$ thienopyridines, 6 $(2.9 \%)$ oral anticoagulation and $8(3.9 \%)$ were not using antithrombotic prophylaxis.

Treatment in the medical therapy arms also varied across studies. In Closure I, patients assigned to medical therapy were treated with warfarin (with a target International Normalised Ratio of 2.0 to 3.0), aspirin (325 mg daily) or both, at the discretion of the principal investigator at each site (further details not provided). In RESPECT, five medical therapies were initially allowed (after randomisation aspirin alone was used in 223 patients $(46.5 \%)$, warfarin alone in $121(46.5 \%)$, clopidogrel alone in 67 patients $(14 \%)$, aspirin with dipyridamole in 39 patients $(8.1 \%)$ and aspirin with clopidogrel in $30(6.2 \%)$. In the PC Trial, antithrombotic treatment was also left to the discretion of the treating physician and could have included antiplatelet therapy or oral anticoagulation (after randomisation, 120 $(57.1 \%)$ of participants were using aspirin, 35 (16.7\%) thienopyridines, $64(30.5 \%)$ oral anticoagulation and $5(2.4 \%)$ were not using antithrombotic prophylaxis.

Adherence to medical therapy/changes in medical therapy was not clearly documented in two of the three studies. In the PC Trial, the percentage of patients using no antithrombotic prophylaxis increased from $2.4 \%$ following randomisation to $7.7 \%$ at 2 years, $11.3 \%$ at 3 years, $11.1 \%$ at 4 years and $12.8 \%$ at 5 years. The distribution of other therapies changed little over 5 years.

A total of $311(13.5 \%)$ participants were lost to follow-up or withdrew consent (range within studies $12-18 \%)$. Loss to follow-up/withdrawal of consent was 


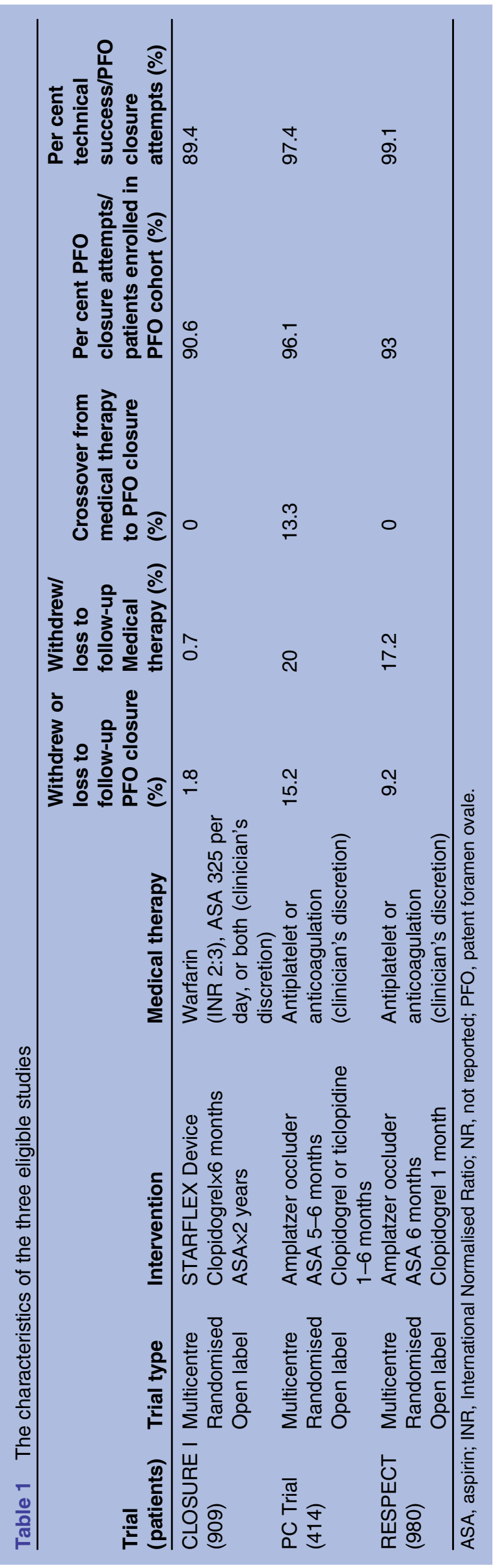

higher in the medical therapy arm $(n=204,18 \%)$ than in the PFO closure arm $(\mathrm{n}=107,9 \%)$. In addition, 14 patients (CLOSURE I=12, PC Trial=1, RESPECT $=1$ ) were demonstrated to have no PFO after randomisation and 11 patients were determined to have no prior history of stroke or TIA (CLOSURE I). Therefore, 336 participants were excluded from our complete case analyses.

The three studies enrolled similar patients (eg, age range from 44.5 to 46 years) with some differences in medical history (table 2). In two of the studies, approximately $70-80 \%$ of patients were enrolled with an index diagnosis of cryptogenic stroke with most of the rest having an index diagnosis of TIA. In one study (RESPECT), all patients enrolled had a diagnosis characterised as stroke but patients with less than $24 \mathrm{~h}$ of symptoms and radiological evidence for infarct were included in this category.

\section{Assessment of risk of bias}

Overall risk of bias was deemed high for all three studies due to missing data (see figure 1) - as noted $13.5 \%$ of participants were lost to follow-up with twice as many lost to follow-up in the medical arm compared with the PFO closure arm. There is also lack of clear description regarding how compliance with medical therapy was assessed-in only one study was medical therapy usage at different time points described.

Participants and study personnel were not blinded in any of the three studies, which likely contributed to differential rates of loss to follow-up. It is unclear whether this would have led to additional bias with respect to the observed outcome rates as a clinical events committee adjudicated events in all three studies.

\section{Outcomes assessment}

\section{Non-fatal ischaemic stroke}

There were a total of 22 non-fatal ischaemic strokes among 1026 patients randomised to PFO closure vs 34 strokes among 941 patients randomised to medical therapy (RR $0.61,95 \%$ CI 0.34 to 1.07 ; heterogeneity: $\mathrm{p}=0.34, \mathrm{I}^{2}=8 \%$, figure 2). Using our best estimate of baseline from the available randomised trials of 52 non-fatal ischaemic strokes over 5 years in 1000 patients treated medically, PFO closure may be associated with 20 fewer strokes per 1000 treated over a period of 5 years (CI 34 fewer to 4 more strokes, low confidence in estimates because of risk of bias and imprecision, table 3).

In a sensitivity analysis including the two studies using the Amplatzer device, $\mathrm{PFO}$ closure was associated with a decreased risk of non-fatal ischaemic stroke (RR 0.44, $95 \% 0.21$ to 0.93 ; heterogeneity: $\mathrm{p}=0.42, \mathrm{I}^{2}=0 \%$ ). In the CLOSURE I study (Starflex device), there was no difference between PFO closure and medical therapy with respect to non-fatal ischaemic stroke (RR $0.87,95 \%$ CI 0.40 to 1.87 ). The test for interaction between these two subset analyses revealed differences consistent with chance $\left(\chi^{2}=1.52, p=0.22\right)$.

We conducted analyses imputing non-fatal strokes for patients excluded from the complete case analysis. In 
Table 2 Characteristics of patients in eligible studies

\begin{tabular}{|c|c|c|c|}
\hline & CLOSURE 1 & RESPECT & PC Trial \\
\hline $\mathrm{N}$ & 909 & 980 & 414 \\
\hline Mean age $\pm S D$ & 46.0 & 45.9 & 44.5 \\
\hline Male (\%) & 51.8 & 54.7 & 49.8 \\
\hline Smoker (\%) & 22.1 & 13.3 & 23.9 \\
\hline \multicolumn{4}{|l|}{ Medical history (\%) } \\
\hline Diabetes & NR & 7.4 & 2.7 \\
\hline Hypertension & 31.0 & 31.4 & 25.8 \\
\hline Hyperlipidaemia & 44.1 & 39.5 & 27.1 \\
\hline Ischaemic heart disease & 1.1 & 2.9 & 1.9 \\
\hline Myocardial infarction & 1.3 & 0.7 & 1 \\
\hline Valvular dysfunction & 10.3 & NR & 3.1 \\
\hline Peripheral vascular disease & 1.3 & 0.6 & 1.2 \\
\hline \multicolumn{4}{|l|}{ Index event (\%) } \\
\hline Stroke & 72 & $100^{*}$ & 79.2 \\
\hline TIA & 28 & 0 & 18.1 \\
\hline Peripheral arterial embolism & 0 & 0 & 2.7 \\
\hline \multicolumn{4}{|l|}{ PFO characteristics (\%) } \\
\hline Moderate or higher shunt & 52.9 & 75.2 & $65.6 \dagger$ \\
\hline Atrial septal aneurysm $>10 \mathrm{~mm}$ & $37.8 \ddagger$ & 35.6 & 23.7 \\
\hline
\end{tabular}

our worst-case analysis (all PFO intervention arm patients excluded from complete case analysis having non-fatal ischaemic stroke, none of participants

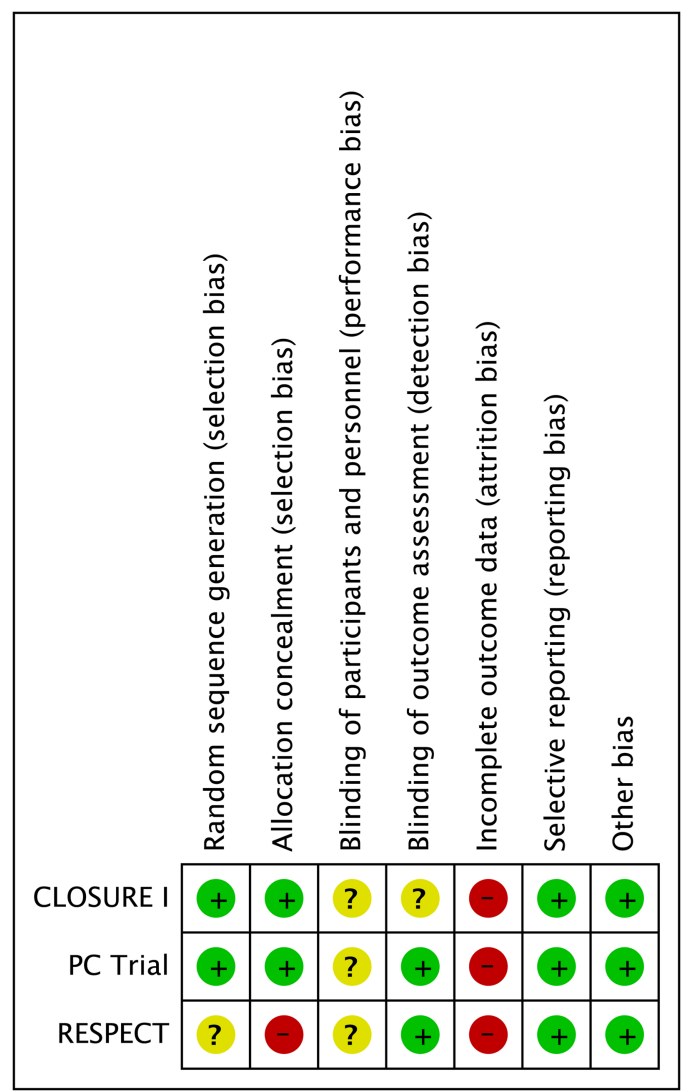

Figure 1 Risk of bias in individual studies. excluded from the medical therapy arm having non-fatal ischaemic stroke), $\mathrm{RR}=4.22,95 \%$ CI 2.93 to 6.08 (heterogeneity: $\mathrm{p}=0.39, \mathrm{I}^{2}=0 \%$ ). In our plausible worst-case analysis in which excluded PFO closure patients had fivefold increased rate of stroke (relative to included participants) and excluded medical therapy patients had a $1 / 5$ rate of stroke (relative to included participants), $\mathrm{PFO}$ closure was associated with an $\mathrm{RR}=0.96,95 \%$ CI 0.56 to 1.66 (heterogeneity: $\mathrm{p}=0.28, \mathrm{I}^{2}=21 \%$ ). Although some might consider the 5:1 ratio we have tested beyond the range of plausibility, there is empirical support for this choice $^{16}$ and our results support rating down confidence in estimates for risk of bias related to missing data.

In the two studies providing per protocol event rates for non-fatal ischaemic stroke there were 18 vs 27 nonfatal ischaemic strokes yielding an RR of 0.66 , $95 \%$ CI 0.32 to 1.38 (heterogeneity: $\mathrm{p}=0.23, \mathrm{I}^{2}=32 \%$ ).

As previous observational studies suggest that patients with cryptogenic stroke and $\mathrm{PFO}$ may be at higher risk for recurrent stroke if they have a concomitant atrial septal aneurysm, we also examined pooled rates of the primary composite endpoint of the studies in this subset. There were 13 events among 378 patients with atrial septal aneurysm undergoing closure compared with 20 events among 380 patients undergoing medical therapy (RR $0.71,95 \%$ CI 0.22 to 2.27 ; heterogeneity: $\left.\mathrm{p}=0.11, \mathrm{I}^{2}=55 \%\right)$.

\section{Transient ischaemic attacks}

Pooling complete case data from the three studies, there were 23 vs 28 TIAs in the PFO closure and medical treatment groups, respectively (RR $0.76,95 \%$ CI 0.44 to 1.32 ; 


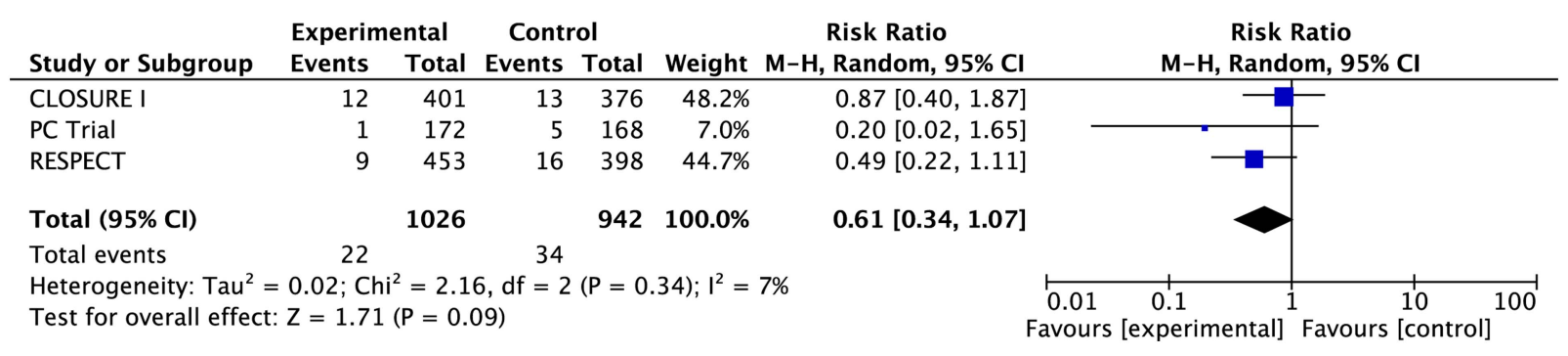

Figure 2 Pooled risk of non-fatal ischaemic stroke with patent foramen ovale closure versus medical therapy.

heterogeneity: $\left.\mathrm{p}=0.64, \mathrm{I}^{2}=0 \%\right)$. PFO closure may be associated with six fewer TIAs over a period of 5 years (CI 15 fewer to 9 more, moderate confidence because of risk of bias (figure 3, table 3).

\section{Total mortality}

There were seven deaths in the PFO closure arm vs 10 deaths in the medical treatment arm of the three studies (RD $-0.00,95 \%$ CI $-0.01,0.01$; heterogeneity: $\mathrm{p}=0.23$, $\left.\mathrm{I}^{2}=31 \%\right)$. None of the deaths were deemed secondary to treatment (PFO closure or antithrombotic therapy) or stroke. PFO closure may have no effect on mortality over a period of 5 years (CI 10 fewer to 10 more, low confidence because of risk of bias and imprecision, table 3 ).

\section{Adverse events}

Pooling data from all three studies, bleeding occurred in 13 vs 7 patients in the PFO closure vs medical treatment arms (all were major bleeds except two bleeds from RESPECT study not classified) (RD 0.00, 95\% CI -0.01 to 0.02 ; heterogeneity $\mathrm{p}=0.12, \mathrm{I}^{2}=53 \%$, see figure 4 ). PFO closure may have no effect on major bleeding over a period of 5 years (CI 10 fewer to 20 more, moderate confidence because of risk of bias, table 3).

Atrial fibrillation occurred in 32 patients undergoing PFO closure vs 8 patients treated with medical therapy (RD 0.02, 95\% -0.02 to 0.06 ; heterogeneity: $\mathrm{p}<0.00001$, $\left.\mathrm{I}^{2}=93 \%\right)$. PFO closure may be associated with 20 more cases of atrial fibrillation per 1000 treated compared with medical therapy over a period of 5 years (CI 20 fewer to 60 more, very low confidence because of risk of bias, inconsistency and imprecision, table 3). Of the 23 cases of atrial fibrillation reported after PFO closure in the CLOSURE I study six were deemed 'sustained'atrial fibrillation in the medical group was not characterised. Of the eight cases of atrial fibrillation in the PC Trial occurring after PFO closure two were transient (in PFO closure arm) and six required cardioversion or were sustained. Atrial fibrillation was not characterised as transient or sustained in the RESPECT study.

We were unable to pool data regarding procedural or device-related complications given differences between studies in reporting styles. Serious procedural or devicerelated adverse events (in addition to bleeding, ischaemic stroke, atrial fibrillation which have already been captured in previous analyses) were reported in 15 patients in the RESPECT trial (3\%). This included eight procedural- related events. Major vascular events related to the procedure occurred in 13 of the 402 patients (3.2\%) in whom PFO closure was attempted in CLOSURE I-these included six major bleeding episodes already captured above. The total number of serious procedural-related adverse events was not specifically reported in the PC Trial although it was noted that no device-related thrombi occurred.

\section{DISCUSSION}

A decade ago, a meta-analysis of observational studies suggested that transcatheter closure of PFO in patients with cryptogenic stroke may prevent more strokes than medical therapy. ${ }^{3}$ The authors noted important limitations in available data and highlighted the need for RCTs to resolve the issue. Since then, thousands of patients have undergone this procedure in a non-RCT setting.

We now have data from three RCTs comparing transcatheter PFO closure to medical therapy in patients with cryptogenic stroke or TIA and PFOs. Our analysis suggests a possible benefit of closure on the major outcome of stroke (RR $0.61,95 \%$ CI 0.34 to 1.07 ). Confidence in the estimate of 20 fewer strokes per 1000 is, however, low, both because of problems with risk of bias and imprecision (CIs include an increase in stroke of 4/1000). Analyses for ischaemic stroke restricted to 'per protocol' patients or patients with concomitant atrial septal aneurysm did not substantially change the observed RRs.

We conducted subgroup analyses evaluating the impact of PFO closure on non-fatal stroke separately in the two studies using the Amplatzer closure device vs the one study using the STARFlex device. Pooled data from the Amplatzer studies suggests that PFO closure may be associated with a decrease in non-fatal ischaemic stroke (RR $0.44,95 \%$ CI 0.21 to 0.93 ), whereas no benefit was observed in the study using the STARFlex device. Although the subgroup hypothesis was made a priori and differences are in the anticipated direction, the analysis is based on between group differences, has not been replicated and differences between results with the two devices is easily explained by chance $(\mathrm{p}=0.22)$. Thus, the subgroup hypothesis has low credibility. ${ }^{17}$

There have been three other meta-analyses. They are limited, however, by failure to fully consider risk of bias issues, failure to use the GRADE approach to determine overall confidence in estimates of intervention effect 
Table 3 GRADE assessment of quality of evidence

\begin{tabular}{|c|c|c|c|c|c|c|c|c|c|}
\hline \multicolumn{6}{|c|}{ Quality assessment } & \multicolumn{4}{|c|}{ Summary of findings } \\
\hline \multirow[b]{2}{*}{$\begin{array}{l}\text { Number of } \\
\text { participants } \\
\text { (studies) }\end{array}$} & \multirow[b]{2}{*}{ Risk of bias } & \multirow[b]{2}{*}{ Inconsistency } & \multirow[b]{2}{*}{ Indirectness } & \multirow[b]{2}{*}{ Imprecision } & \multirow[b]{2}{*}{$\begin{array}{l}\text { Publication } \\
\text { bias }\end{array}$} & \multirow{2}{*}{$\begin{array}{l}\text { Relative } \\
\text { effect } \\
\text { or risk } \\
\text { difference } \\
(95 \% \mathrm{Cl})\end{array}$} & \multicolumn{2}{|c|}{$\begin{array}{l}\text { Anticipated absolute effects } \\
\text { Time frame: } 5 \text { years }\end{array}$} & \multirow[b]{2}{*}{ Quality of evidence } \\
\hline & & & & & & & $\begin{array}{l}\text { Risk with } \\
\text { medical } \\
\text { therapy }\end{array}$ & $\begin{array}{l}\text { Risk difference } \\
\text { with PFO closure } \\
(95 \% \mathrm{Cl})\end{array}$ & \\
\hline \multicolumn{10}{|c|}{ Non-fatal ischaemic stroke (critical outcome) } \\
\hline $\begin{array}{l}1968 \\
\text { (3 RCTs) }\end{array}$ & $\begin{array}{l}\text { Serious } \\
\text { limitations }^{*}\end{array}$ & $\begin{array}{l}\text { No serious } \\
\text { limitations }\end{array}$ & $\begin{array}{l}\text { No serious } \\
\text { limitations }\end{array}$ & $\begin{array}{l}\text { Imprecise } \\
\mathrm{Cl} \text { includes benefit } \\
\text { and no effect }\end{array}$ & Undetected & $\begin{array}{l}R R 0.61 \\
(0.34 \text { to } 1.07)\end{array}$ & $52 / 1000+$ & $\begin{array}{l}20 \text { fewer per } 1000 \\
\text { (from } 34 \text { fewer to } 4 \\
\text { more) }\end{array}$ & $\begin{array}{l}\oplus \oplus \mathrm{OO} \\
L O W \text { due to risk of bias and } \\
\text { imprecision }\end{array}$ \\
\hline \multicolumn{10}{|c|}{ TIA (important outcome) } \\
\hline $\begin{array}{l}1968 \\
\text { (3 RCTs) }\end{array}$ & $\begin{array}{l}\text { Serious } \\
\text { limitations }^{*}\end{array}$ & $\begin{array}{l}\text { No serious } \\
\text { limitations }\end{array}$ & $\begin{array}{l}\text { No serious } \\
\text { limitations }\end{array}$ & $\begin{array}{l}\text { No serious } \\
\text { limitations } \ddagger\end{array}$ & Undetected & $\begin{array}{l}R R 0.76 \\
\text { (0.44 to } 1.32)\end{array}$ & $27 / 1000 \S$ & $\begin{array}{l}6 \text { fewer per } 1000 \\
\text { (from } 15 \text { fewer to } 9 \\
\text { more) }\end{array}$ & $\begin{array}{l}\oplus \oplus \oplus \mathrm{O} \\
M O D E R A T E \text { due to risk of bias }\end{array}$ \\
\hline \multicolumn{10}{|c|}{ Total mortality (critical outcome) } \\
\hline 1968 (3 RCTs) & $\begin{array}{l}\text { Serious } \\
\text { limitations }^{*}\end{array}$ & $\begin{array}{l}\text { No serious } \\
\text { limitations }\end{array}$ & $\begin{array}{l}\text { No serious } \\
\text { limitations }\end{array}$ & $\begin{array}{l}\text { Imprecise } \\
\mathrm{Cl} \text { includes benefit } \\
\text { and harm }\end{array}$ & Undetected & $\begin{array}{l}R D 0.00 \\
(-0.01,0.01)\end{array}$ & $15 / 1000^{\star \star}$ & $\begin{array}{l}\text { O fewer per } 1000 \\
\text { (from } 10 \text { fewer to } \\
10 \text { more) }\end{array}$ & $\begin{array}{l}\oplus \oplus \mathrm{OO} \\
L O W \text { due to risk of bias and } \\
\text { imprecision }\end{array}$ \\
\hline \multicolumn{10}{|c|}{ Major bleeding (important outcome) } \\
\hline $\begin{array}{l}2254 \\
\text { (3 RCTs) }\end{array}$ & $\begin{array}{l}\text { Serious } \\
\text { limitations }^{*}\end{array}$ & $\begin{array}{l}\text { No serious } \\
\text { inconsistency }\end{array}$ & $\begin{array}{l}\text { No serious } \\
\text { limitationsł }\end{array}$ & $\begin{array}{l}\text { No serious } \\
\text { limitations }\end{array}$ & Undetected & $\begin{array}{l}R D 0.00 \\
(-0.01,0.02)\end{array}$ & $7 / 1000+\dagger$ & $\begin{array}{l}0 \text { more per } 1000 \\
(10 \text { fewer to } 20 \\
\text { more) }\end{array}$ & $\begin{array}{l}\oplus \oplus \oplus \mathrm{O} \\
M O D E R A T E \text { due to risk of bias }\end{array}$ \\
\hline \multicolumn{10}{|c|}{ Atrial fibrillation (important outcome) $\ddagger \ddagger$} \\
\hline $\begin{array}{l}2254 \\
\text { (3 RCTs) }\end{array}$ & $\begin{array}{l}\text { Serious } \\
\text { limitations }^{*}\end{array}$ & $\begin{array}{l}\text { Serious } \\
\text { inconsistency§§ }\end{array}$ & $\begin{array}{l}\text { No serious } \\
\text { limitations }\end{array}$ & $\begin{array}{l}\text { Imprecise } \\
\mathrm{Cl} \text { includes benefit } \\
\text { and harm }\end{array}$ & Undetected & $\begin{array}{l}R D 0.02 \\
(-0.02,0.06)\end{array}$ & 12/1000ㄲ & $\begin{array}{l}20 \text { more per } 1000 \\
(20 \text { fewer to } 60 \\
\text { more) }\end{array}$ & $\begin{array}{l}\oplus \mathrm{OOO} \\
\text { VERY LOW due to risk of bias, } \\
\text { inconsistency and imprecision }\end{array}$ \\
\hline
\end{tabular}

*Serious risk of bias due to substantial loss to follow-up in each of the three studies; loss to follow-up greater in medical therapy arms. See text for other potential sources of bias in individual

thadies.
†Bane rate derived from pooled RESPECT and PC Trial data- 21 non-fatal ischaemic strokes detected in medical therapy arm over a total of 2019 patient-years $\times 1000 \times 5$ years.

†Although $\mathrm{Cl}$ includes benefit and harm, magnitude of extremes for this type of outcome deemed too low to appreciably impact patient decision-making.

$\S$ Baseline rate derived from pooled RESPECT and PC Trial data-11 TIAs detected in medical therapy arm over a total of 2019 patient-years $\times 1000 \times 5$ years.

१None of deaths due to stroke, treatment related bleeding, or device implantation.

${ }_{\star \star *}$ Baseline rate derived from pooled RESPECT and PC Trial data -6 cases of total mortality detected in medical therapy arm over a total of 2019 patient years $\times 1000 \times 5$ years.

††Baseline rate derived from pooled RESPECT and PC Trial data- 3 cases of major bleeding detected in medical therapy arm over a total of 2019 patient-years $\times 1000 \times 5$ years.

¥¥Type of atrial fibrillation (transient vs sustained) not reported in medical therapy arms or in PFO closure arm of RESPECT study. Of 31 cases of atrial fibrillation in the remaining 2 studies

19 were characterised as transient.

$\left.\S \S\right|^{2}=93 \%, \mathrm{p} \leq 0.00001$

Iq⿴囗Baseline rate derived from pooled RESPECT and PC Trial data-five cases of atrial fibrillation detected in the medical therapy arm over a total of 2019 patient-years $\times 1000 \times 5$ years.

$\mathrm{PFO}$, patent foramen ovale; RD, risk difference; RR, risk ratio; RCT, randomised controlled trial; TIA, transient ischaemic attack. 


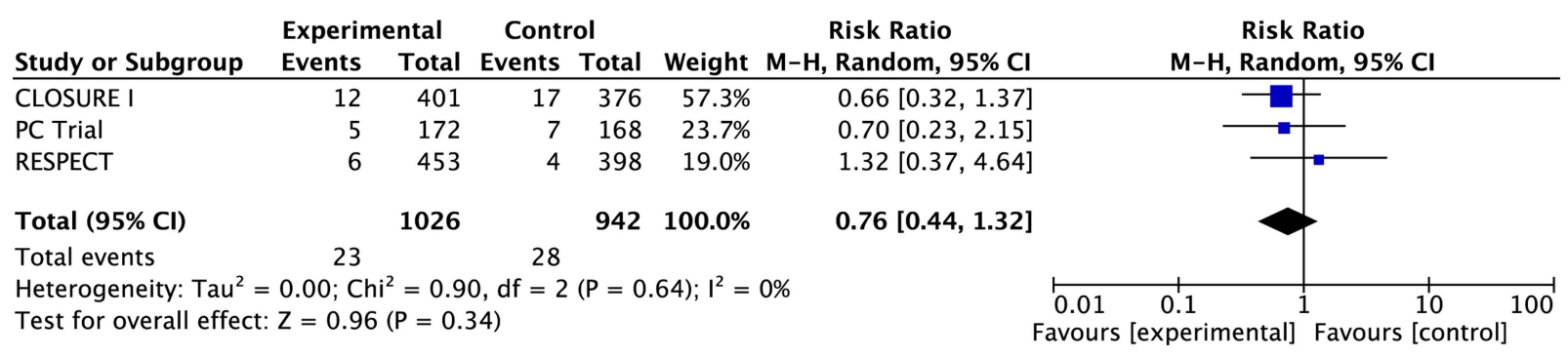

Figure 3 Pooled risk of transient ischaemic attack with patent foramen ovale closure versus medical therapy.

and failure to consider the limitations of composite endpoints. ${ }^{18-20}$ In the most recent of these analyses, PFO closure was associated with an effect-estimate HR of 0.67 (95\% CI 0.44 to 1.00 ) for the prevention of 'neurological events'. However, it appears that this composite endpoint included the softer endpoint of TIA in addition to stroke and mortality.

It is possible that a larger sample size and more rigorously performed studies would definitively identify an important benefit in the total patient population, or in a subgroup. Our review demonstrates, however, that such additional studies may also fail to demonstrate benefit or in comparison to effective antithrombotic prophylaxis, may demonstrate an increase in strokes.

Although some concern arises from the possible lack of concealment of randomisation in one study and the apparent failure to blind outcome adjudication in another, the major problem in terms of risk of bias is the high loss to follow-up in these studies and the twofold greater loss to follow-up in patients in the medical therapy arms than the PFO closure arms (overall 9\% in PFO and $18 \%$ in the medical therapy arms).

Our primary analysis was restricted to patients with available data (complete case analysis). If event rates differed in those with missing data in intervention and control groups-of particular concern would be higher rates of events in those lost to follow-up in the PFO closure arm than the medical therapy arm-the complete case results may be misleading. In an additional analysis in which patients lost to follow-up in the PFO arm were assumed to have fivefold increased risk of stroke and those lost to follow-up in the medical therapy arm had a fivefold decreased risk of stroke, there was no longer a trend favouring PFO closure (RR 0.96). This finding supports our rating down confidence in estimates of effect for risk of bias.
Another issue is the rigour with which control arm clinicians encouraged compliance with antithrombotic prophylaxis in medical patients. In two of the studies, dose and type of antithrombotic therapy in the medical therapy arm were left to the treating physician's discretion. Only one of the studies reported adherence and/or changes over time in medical therapy in both arms. Leaving therapy in the medical arm to the physician's discretion could be considered to represent 'usual care' for those randomised to medical therapy. Usual care may, however, change over time and differ in the jurisdictions in which the trial is conducted in comparison to other jurisdictions. Patients and clinicians may, therefore, be more interested in the effect of $\mathrm{PFO}$ closure versus a particular antithrombotic regimen with a high level of adherence. Unfortunately, there have been no RCTs adequately comparing specific antiplatelet or antithrombotic therapies for this indication.

Stroke occurring due to paradoxical emboli through a $\mathrm{PFO}$ results from thrombi originating in the venous circulation or perhaps from the associated atrial septal aneurysm itself. $^{21}{ }^{22}$ Warfarin has been shown to be more effective than antiplatelet therapy for the treatment and secondary prevention of venous thromboembolic events. Observational studies suggest that oral anticoagulation is superior to aspirin for the prevention of stroke in patients with PFO albeit with increased bleeding. ${ }^{23}{ }^{24}$ In the Patent Foramen Ovale in Cryptogenic Stroke Study (substudy of the randomised Warfarin-Aspirin Recurrent Stroke Study) there were 98 patients with cryptogenic stroke and PFO-42 were randomised to warfarin and 56 received aspirin. ${ }^{25}$ Two-year rates of recurrent stroke were lower in patients receiving warfarin $(9.5 \%$ vs $17.9 \%$, ) but chance easily explains this $(\mathrm{p}=0.28)$.

Given the uncertainty of the optimal antithrombotic regimen, subsequent trials must give this issue careful

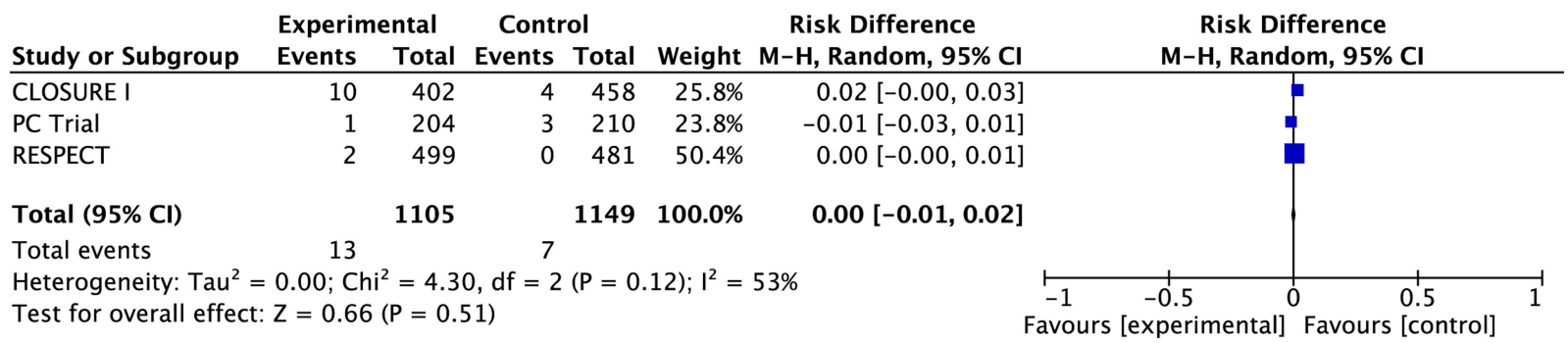

Figure 4 Pooled risk of major bleeding with patent foramen ovale closure versus medical therapy. 
thought. One option for the medical arm would be careful exploration of individual patient values and preferences. Patients highly averse to bleeding risk and the burdens of anticoagulant therapy could receive only an antiplatelet agent, whereas those less bleeding and burden averse could receive an anticoagulant. Use of an oral anticoagulant rather than warfarin in those choosing anticoagulation would be a possibility. Such an approach might represent optimal medical care, perhaps the appropriate comparator to PFO closure. Another option would be three-arm study with both antiplatelet and anticoagulant arms.

We conclude that the available data warrants only low confidence in the impact of PFO versus medical therapy. Thus, additional RCTs are still required-two such studies are listed as actively recruiting on the $\mathrm{NIH}$ website ClinicalTrials.gov. Ideally, when pooled across studies, sample sizes will be large enough to definitively establish the impact of PFO closure versus medical therapy on the most important outcome, ischaemic stroke. As important, results will be more compelling if the ongoing studies have implemented successful strategies to ensure complete or near-complete follow-up and have paid careful attention to decisions regarding medical prophylaxis and optimising adherence in both arms of the study. In the interval, patients should be made aware of the management options and the uncertainty underlying their effectiveness.

Contributors FS contributed to the conception and design of the study, the acquisition, analysis and interpretation of the data, drafting the work, and gave the final approval of the version to be published. LCL and SK contributed to the design of the study, the acquisition, analysis and interpretation of the data, critical revision of the work for important intellectual content, and gave final approval of the version to be published. GG contributed to the design of the study, the acquisition, analysis and interpretation of the data, drafting and critical revision of the work for important intellectual content, and gave final approval of the version to be published.

Funding This research received no specific grant from any funding agency in the public, commercial or not-for-profit sectors.

Competing interests None.

Provenance and peer review Not commissioned; externally peer reviewed.

Data sharing statement No additional data are available.

Open Access This is an Open Access article distributed in accordance with the Creative Commons Attribution Non Commercial (CC BY-NC 3.0) license, which permits others to distribute, remix, adapt, build upon this work noncommercially, and license their derivative works on different terms, provided the original work is properly cited and the use is non-commercial. See: http:// creativecommons.org/licenses/by-nc/3.0/

\section{REFERENCES}

1. Webster MW, Chancellor AM, Smith $\mathrm{HJ}$, et al. Patent foramen ovale in young stroke patients. Lancet 1988;2:11-12.

2. Lechat $\mathrm{P}$, Mas JL, Lascault $\mathrm{G}$, et al. Prevalence of patent foramen ovale in patients with stroke. N Engl J Med 1988;318:1148-52.
3. Khairy P, O'Donnell CP, Landzberg MJ. Transcatheter closure versus medical therapy of patent foramen ovale and presumed paradoxical thromboemboli: a systematic review. Ann Intern Med 2003;139:753-60.

4. Furlan AJ, Reisman M, Massaro J, et al. Closure or medical therapy for cryptogenic stroke with patent foramen ovale. N Engl J Med 2012;366:991-9.

5. Carroll JD, Saver JL, Thaler DE, et al. Closure of patent foramen ovale versus medical therapy after cryptogenic stroke. $N$ Engl J Med 2013;368:1092-100.

6. Meier B, Kalesan B, Mattle HP, et al. Percutaneous closure of patent foramen ovale in cryptogenic embolism. N Engl J Med 2013;368:1083-91.

7. Higgins JP, Altman DG, Gotzsche PC, et al. The Cochrane Collaboration's tool for assessing risk of bias in randomised trials. BMJ 2011;343:d5928.

8. Guyatt $\mathrm{GH}$, Oxman AD, Vist GE, et al. GRADE: an emerging consensus on rating quality of evidence and strength of recommendations. BMJ 2008;336:924-6.

9. Guyatt GH, Oxman AD, Vist G, et al. GRADE guidelines: 4. Rating the quality of evidence-study limitations (risk of bias). J Clin Epidemiol 2011;64:407-15.

10. Guyatt $\mathrm{GH}$, Oxman $A D$, Kunz R, et al. GRADE guidelines 6 . Rating the quality of evidence-imprecision. J Clin Epidemiol 2011;64:1283-93.

11. Guyatt GH, Oxman AD, Kunz R, et al. GRADE guidelines: 7. Rating the quality of evidence-inconsistency. J Clin Epidemiol 2011;64:1294-302.

12. Guyatt GH, Oxman AD, Kunz R, et al. GRADE guidelines: 8. Rating the quality of evidence -indirectness. J Clin Epidemiol 2011;64:1303-10.

13. Guyatt GH, Oxman AD, Montori V, et al. GRADE guidelines: 5. Rating the quality of evidence-publication bias. J Clin Epidemiol 2011;64:1277-82.

14. Akl EA, Briel M, You JJ, et al. Potential impact on estimated treatment effects of information lost to follow-up in randomised controlled trials (LOST-IT): systematic review. BMJ 2012;344:e2809.

15. Murad MH, Montori VM, Walter SD, et al. Estimating risk difference from relative association measures in meta-analysis can infrequently pose interpretational challenges. J Clin Epidemiol 2009;62:865-7.

16. Geng EH, Emenyonu N, Bwana MB, et al. Sampling-based approach to determining outcomes of patients lost to follow-up in antiretroviral therapy scale-up programs in Africa. JAMA 2008;300:506-7.

17. Sun X, Briel M, Walter SD, et al. Is a subgroup effect believable? Updating criteria to evaluate the credibility of subgroup analyses. BMJ 2010;340:c117.

18. Pineda AM, Nascimento FO, Yang SC, et al. A meta-analysis of transcatheter closure of patent foramen ovale versus medical therapy for prevention of recurrent thromboembolic events in patients with cryptogenic cerebrovascular events. Catheter Cardiovasc Interv 2013;82:968-75.

19. Kitsios GD, Thaler DE, Kent DM. Potentially large yet uncertain benefits: a meta-analysis of patent foramen ovale closure trials. Stroke 2013;44:2640-3.

20. Khan AR, Bin Abdulhak AA, Sheikh MA, et al. Device closure of patent foramen ovale versus medical therapy in cryptogenic stroke: a systematic review and meta-analysis. JACC Cardiovas Interv 2013:6:1316-23.

21. Aryal MR, Pradhan R, Pandit AA, et al. A 'teapot' atrial septal aneurysm with spontaneous thrombus in an asymptomatic patient. Circulation 2013;128:e409-10.

22. Schneider B, Hanrath P, Vogel P, et al. Improved morphologic characterization of atrial septal aneurysm by transesophageal echocardiography: relation to cerebrovascular events. J AmColl Cardiol 1990;16:1000-9.

23. Cujec B, Mainra R, Johnson DH. Prevention of recurrent cerebral ischemic events in patients with patent foramen ovale and cryptogenic strokes or transient ischemic attacks. Can J Cardiol 1999;15:57-64.

24. Orgera MA, O'Malley PG, Taylor AJ. Secondary prevention of cerebral ischemia in patent foramen ovale: systematic review and meta-analysis. South Med J 2001;94:699-703.

25. Homma S, Sacco RL, Di Tullio MR, et al. Effect of medical treatment in stroke patients with patent foramen ovale: patent foramen ovale in Cryptogenic Stroke Study. Circulation 2002;105:2625-31. 